\title{
Erratum: Pressure and energy of compressional shocks in two-dimensional Yukawa systems [Phys. Rev. E 100, 043203 (2019)]
}

Wei Lin, M. S. Murillo, and Yan Feng (D)

(Q) (Received 2 January 2020; published 21 January 2020)

DOI: 10.1103/PhysRevE.101.019902

The color bar of Fig. 3 in the original paper should be multiplied by $10^{-4}$, instead of $10^{-5}$ in our paper. We regret this typographical error.

None of the reported data in the original paper are affected, and likewise, the conclusions in the original paper are not affected. 